\title{
Comparing the Performance of Calf Circumference, Albumin, and BMl for Predicting Mortality in Immobile Patients
}

\author{
Xiao-Ming Zhang' \\ Xinjuan Wu' \\ Yunfen $\mathrm{Ma}{ }^{\mathrm{I}}$ \\ Chen Zhu' \\ Jing Cao' \\ Ge Liu' \\ Fang-Fang Li \\ Andy SK Cheng $\mathbb{D}^{2}$ \\ 'Department of Nursing, Chinese \\ Academy of Medical Sciences - Peking \\ Union Medical College, Peking Union \\ Medical College Hospital (Dongdan \\ Campus), Beijing, 100730, People's \\ Republic of China; ${ }^{2}$ Department of \\ Rehabilitation Sciences, The Hong Kong \\ Polytechnic University, Hong Kong, \\ People's Republic of China
}

Background: Immobile patients often suffer from malnutrition and low calf circumference (CC), a potential surrogate marker of low muscle mass, which has been widely explored as a link with mortality among patients with inconsistent results. In addition, studies comparing CC, BMI, and albumin to predict mortality among immobile patients are limited. The aim of our study was to compare the performance of these three parameters for predicting mortality among immobile patients.

Methods: This is a secondary analysis of our previous study consisting of immobile patients, age $>18$ years. Data were collected between November 2015 and March 2016 by trained nurses through a case report form (CRF) that included clinical data and sociodemographic variables. In addition, anthropometric parameters, including body weight, height and calf circumference, were also measured. The outcome of our study was mortality with follow-up length being 90 days. Multivariate linear and logistic regression analysis were adopted to identify the association between $\mathrm{CC}$ and mortality, and a receiver operating characteristic curve analysis was also used to compare the performance of CC, BMI, and albumin.

Results: Of the 387 patients who were included (51.42\% male), with an average age of 61.22 $(\mathrm{SD}=15.18)$, the prevalence of mortality was $5.43 \%$. The results showed that, after full adjustment, calf circumference was a protective factor for reducing the risk of mortality $(\mathrm{OR}=0.79 ; 95 \%$ CI:0.65-0.96). Meanwhile, immobile patients with low calf circumference had an increased risk of mortality, compared to those whose condition was normal (OR=4.24; 95\%CI:1.07-16.74). Receiver operating characteristic curve analysis showed that $\mathrm{CC}$ combined with albumin (AUC $=0.812 ; 95 \% \mathrm{CI}=0.733-0.890$ ) had the highest $\mathrm{AUC}$ value compared to calf circumference (AUC $=0.759 ; 95 \% \mathrm{CI}: 0.662-0.856)$, BMI (AUC $=0.653 ; 95 \% \mathrm{CI}: 0.538-0.767)$ and albumin (AUC $=0.735 ; 95 \%$ CI:0.638-0.832), respectively, in predicting mortality in immobile patients.

Conclusion: The relationship between calf circumference and mortality was significantly negative and low calf circumference was associated with a high risk of 90-day mortality, compared to those with normal calf circumference. The combined calf circumference with albumin had good discrimination in predicting mortality among immobile patients. Therefore, it can routinely be used in a clinical setting by medical staff to predict mortality in immobile patients, as it is easy to obtain.

Keywords: calf circumference, immobile patients, albumin, mortality

\section{Background}

The number of hospital inpatients, especially older inpatients, has increased sharply in recent years due to a progressively aging society. Inpatients often suffer from malnutrition because of a typically significant disease burden and other geriatric 
syndromes, ${ }^{1}$ which is a particular consideration for immobile patients. Bed rest and immobility are important risk factors for malnutrition $(\mathrm{OR}=5.88,95 \% \mathrm{CI}: 2.25-15.4) .{ }^{2} \mathrm{It}$ is estimated that the prevalence of malnutrition among hospitalized patients ranges from $21 \%-54 \%$, rising to $60 \%$ among older adults. ${ }^{3,4}$ Malnutrition exerts an increased impact on adverse outcomes, including mortality, morbidity, ${ }^{5}$ disability, and low quality of life. ${ }^{6,7}$ Therefore, identifying the risk factors for malnutrition and carrying out corresponding interventions is essential to reduce mortality rates. The new criteria of malnutrition, based on the Global Leadership Initiative on Malnutrition (GLIM) and sponsored by several academic organizations, require at least one phenotypic and one etiologic criterion. ${ }^{8}$ Phenotypes consist of three criteria: unintentional weight loss, declining muscle loss or low BMI. Among these three criteria, muscle mass is very important because it is a core component of maintaining muscle structure and function, to some extent reflecting patient nutritional status, ${ }^{9}$ which is also an essential element to define sarcopenia by low muscle mass. ${ }^{10}$ Inpatients experiencing short or long periods of immobilization, usually due to mobility limitations or disease recovery requirements, often suffered from loss of skeletal muscle mass, eventually increasing the risk of malnutrition. ${ }^{11}$ There are several methods to assess patient muscle mass in a clinical routine. The common standards are MRI or CT or dual-energy X-ray assessment (DXA), plus bioimpedance analysis (BIA) to measure muscle mass and structure in a community setting. ${ }^{8,12}$ Previously published studies reported that patients with low muscle mass were at increased risk of mortality, compared to those in normal condition, when using different measurement methods. ${ }^{13,14}$ However, these methods have some disadvantages, such as lack of convenience, as well as requiring specialized equipment and being time-consuming and expensive, possibly limiting their application in a clinical setting. ${ }^{15,16}$ Exploring new surrogate measures to accurately reflect muscle mass is urgent.

Currently, anthropometric parameters, such as calf circumference ( $\mathrm{CC})$, which has been recommended by the revised European consensus on the definition and diagnosis for assessing muscle mass, were used to assess nutritional status. $^{12,17}$ It is demonstrated that $\mathrm{CC}$ has good discrimination and was validated when compared with DXA for detecting muscle mass. ${ }^{18}$ The association between $\mathrm{CC}$ and mortality has been widely explored in several studies. ${ }^{19-30}$ Sousa et al $^{19}$ reported that low CC was a predictor for mortality among cancer patients, with the hazard ratio figure being 3.01 (95\%CI:1.52-5.98). de Sousa et $\mathrm{al}^{26}$ also reported similar results among older adults with Alzheimer's disease. Whereas, recently, Aliberti et $\mathrm{al}^{31}$ reported that low muscle mass assessed by $\mathrm{CC}$ ( $<33 \mathrm{~cm}$ for women or $<34 \mathrm{~cm}$ for men) was not associated with an increased risk of mortality. A similar study was conducted by Tarnowski et al, ${ }^{24}$ who reported that low CC did not significantly increase the risk of mortality among emergency patients. Given these controversial results, there is a need for more studies to explore this issue. In addition, there are few studies comparing the discrimination performances between the different nutritional parameters, BMI, albumin, and CC, for predicting mortality in immobile patients. Therefore, our study has two objectives: (1) to explore the association between CC and mortality among immobile inpatients. (2) to compare discrimination performance between BMI, albumin, and $\mathrm{CC}$ for predicting mortality and to develop a good predictive model for mortality in patients with immobility. We hypothesized that exploring and comparing the performance of these nutritional parameters can assist clinicians in establishing a predictive system that stratifies immobile patient groups at high risk of mortality, and help guide clinicians in performing early effective interventions.

\section{Methods}

This was a secondary analysis of a cohort study derived from our previously published study ${ }^{32}$ that explores the influencing factors of pressure injuries among immobile inpatients.

\section{Participants}

Twenty-five public general hospitals were contacted from November 2015 to March 2016. The inclusion criteria required that participants (1) were immobile inpatients aged 18 years or older, and (2) needed to be immobilized due to disease, and sedation after surgery. Participants were excluded if they were unconscious and/or unable to provide informed consent. As a secondary analysis, we only analyzed participants who had completed an assessment of CC within 24 hours after hospital admission. According to our previous study, the definition of immobility was a confirmation that patients had to conduct most of their basic physiological needs at their bedside, with the exception of medical examination with active or passive bedside sitting or standing. After screening, 387 participants from 13 hospitals were included in this 
study. Table S1 summarizes the initial 25 hospitals and total sample contacted, and Table S2 provides the detailed information of 13 hospitals and 387 participants in this present study.

\section{Data Collection}

Eligible participants were enrolled in our study by trained nurses at each hospital. Characteristics, including clinical and sociodemographic variables, were collected by trained researchers via case report form (CRF). Sociodemographic variables included gender, age, education, ethnicity, and smoking. In addition, clinical variables consisted of red and white blood cell count, hemoglobin, neutrophils, leukomonocyte, fasting plasma glucose level, pneumonia, bedsores, urinary tract infection, surgical operation, primary diagnosis and bed-rest days. We also recorded the Charlson Comorbidity Index, which was used to categorize comorbidities of patients according to the international classification of disease diagnosis. Furthermore, anthropometric parameters, including body weight, height, and $\mathrm{CC}$, were also measured by trained nurses 24 hours after admission. BMI is equal to body weight divided by the square of height. For $\mathrm{CC}$ measurements, well-trained nurses followed a standard procedure to measure $\mathrm{CC}$ using inextensible tape when the patient lay down on the bed in the supine and relaxed position. Patients were measured in both the left and right leg. We recorded both values and calculated the average of both values for statistical analysis. In this study, calf circumference was measured as both a continuous variable with $0.1 \mathrm{~cm}$ intervals, and also categorized into low $\mathrm{CC}$ and normal $\mathrm{CC}$ with the cutoff value at $31 \mathrm{~cm}^{33}$

\section{Outcomes}

The outcome in our study was mortality, including during hospital stay or follow-up three months after enrollment. When patients died in the hospital, we obtained this information through the hospital case management system; otherwise, we used cell phone interviews to obtain it.

\section{Ethical Considerations}

As reported before, this study was approved by the ethics review boards of 25 cooperating hospitals in China (S-700). Written informed consent was obtained after all patients agreed to enroll.

\section{Data Analysis}

The data were displayed as categorical and continuous variables with frequency or proportion and mean (standard deviation) or median (IQR), respectively. We used Student's $t$-test or Mann-Whitney tests and Chi-square or Fisher's Exact tests, if necessary, to compare the differences between two groups (low $\mathrm{CC}$ versus normal CC; survivors versus non-survivors). Multivariate linear and logistic regression was also used to identify the association between nutritional variables (CC, BMI and albumin) and mortality. We also listed different models by adjusting different parameters. Model 1 was unadjusted; Model 2 adjusted age, gender and education; Model 3 adjusted Model 2 plus included smoking, red and white blood cell count, hemoglobin, neutrophils, leukomonocyte, pneumonia, urinary tract infection, Charlson Comorbidity Index $(\geq 2)$, antibiotics, glucocorticoids and surgery. Meanwhile, we used a generalized additive model (GAM) and smooth curve fitting analysis to detect whether there was a nonlinear relationship between calf circumference and 90-day mortality. A receiver operating characteristic curve analysis was employed to compare discrimination performance between $\mathrm{CC}$, BMI, albumin, and CC combined with albumin. All analysis was performed using statistical software packages $\mathrm{R}$ and EmpowerStats software, with a significant difference in cutoff value at less than 0.05 .

\section{Results}

\section{Clinical Characteristics in Two Groups (C $C \geq 3 \mathrm{Icm}$ versus $\mathrm{CC}<3 \mathrm{Icm}$ )}

Table 1 describes the baseline characteristics of our study. There were 387 participants $(51.42 \%$ male) who were included, with an average age of $61.22(\mathrm{SD}=15.18)$; the prevalence of 90 -day mortality was $5.43 \%$. Only $13.18 \%$ were current smokers, and the majority of the sample was non-smoking, with $9.3 \%$ being past smokers. Of this population, $116(30.61 \%)$ participants had a primary diagnosis of orthopedic disorders and fractures, $41(10.82 \%)$ participants had suffered a stroke and 29 participants had cancer. A total of 140 (36.18\%) participants had a higher Charlson Comorbidity Index score, and the median of bedrest day in this study population was 11.00 (5.00-18.50). The average BMI was $23.98 \mathrm{~kg} / \mathrm{m}^{2}$; albumin was $35.38 \mathrm{~g} / 1$; and $\mathrm{CC}$ was $32.57 \mathrm{~cm}$. Only a small percentage of the sample had a urinary tract infection $(2.84 \%)$, and a minority of patients had bedsores (4.65\%), with $19.64 \%$ who had pneumonia. The distribution of education 
Table I Subject Clinical Characteristics

\begin{tabular}{|c|c|c|c|c|c|}
\hline Calf Circumference & Overall & $\mathrm{CC} \geq 3 \mathrm{I} \mathrm{cm}$ & $\mathrm{CC}<3 \mathrm{Icm}$ & Standardize Diff. & $P$-value \\
\hline $\mathrm{N}$ & 387 & $264(68.2 \%)$ & $123(3 \mid .8 \%)$ & & \\
\hline Age (SD, years) & $61.22(15.18)$ & $59.22(14.66)$ & $65.50(15.44)$ & $0.42(0.20,0.63)$ & $<0.001$ \\
\hline Albumin (SD, g/L) & $35.38(6.00)$ & $35.98(5.83)$ & $34.15(6.18)$ & $0.30(0.08,0.53)$ & 0.001 \\
\hline BMI (SD, kg/m²) & $23.98(3.55)$ & $24.92(3.18)$ & $21.96(3.46)$ & $0.89(0.67,1.11)$ & $<0.001$ \\
\hline Red blood cell (SD, I0^9/L) & $3.91(1.58)$ & $3.93(0.75)$ & $3.87(2.57)$ & $0.03(-0.19,0.25)$ & 0.746 \\
\hline Hemoglobin (g/L, SD) & II 5.90 (22.78) & II $8.74(23.31)$ & $109.98(20.48)$ & $0.40(0.18,0.62)$ & $<0.001$ \\
\hline White blood cell (SD, I0^9/L) & $9.66(4.33)$ & $9.60(4.42)$ & $9.77(4.14)$ & $0.04(-0.18,0.26)$ & 0.549 \\
\hline Neutrophils (SD, I0^9/L) & $7.57(4.14)$ & $7.44(4.21)$ & $7.83(3.99)$ & $0.09(-0.12,0.31)$ & 0.286 \\
\hline Leukomonocyte (SD, I0^9/L) & $1.29(0.92)$ & $1.33(1.01)$ & $1.20(0.69)$ & $0.14(-0.08,0.36)$ & 0.214 \\
\hline Fasting plasma glucose (SD, mmol/L) & $6.85(2.5 \mathrm{I})$ & $6.74(2.45)$ & $7.10(2.64)$ & $0.14(-0.11,0.40)$ & 0.101 \\
\hline Calf circumference (SD, $\mathrm{cm})$ & $32.57(3.96)$ & $34.49(3.12)$ & $28.46(1.91)$ & $2.33(2.06,2.60)$ & $<0.001$ \\
\hline Gender (n, \%) & & & & $0.30(0.08,0.5 \mathrm{I})$ & 0.007 \\
\hline Male & 199 (5I.42\%) & I 48 (56.06\%) & $5 I(4 \mid .46 \%)$ & & \\
\hline Female & 188 (48.58\%) & 116 (43.94\%) & $72(58.54 \%)$ & & \\
\hline Ethnicity (n, \%) & & & & $0.10(-0.11,0.32)$ & 0.372 \\
\hline Han & 369 (66.24\%) & $250(94.70 \%)$ & 119 (96.75\%) & & \\
\hline Other & $188(33.76$ & 14 (5.30\%) & $4(3.25 \%)$ & & \\
\hline Education (n, \%) & & & & $0.45(0.23,0.66)$ & $<0.001$ \\
\hline Illiterate & 49 (12.66\%) & 22 (8.33\%) & 27 (21.95\%) & & \\
\hline Primary & 107 (27.65\%) & $69(26.14 \%)$ & $38(30.89 \%)$ & & \\
\hline Middle school and above & I7I (44.19\%) & $129(48.86 \%)$ & $42(34.15 \%)$ & & \\
\hline College degree or above & $60(15.50 \%)$ & $44(16.67 \%)$ & $16(13.01 \%)$ & & \\
\hline Bedsores (n, \%) & & & & $0.16(-0.05,0.38)$ & 0.158 \\
\hline No & 369 (95.35\%) & $249(94.32 \%)$ & $120(97.56 \%)$ & & \\
\hline Yes & $18(4.65 \%)$ & 15 (5.68\%) & $3(2.44 \%)$ & & \\
\hline Pneumonia $(\mathrm{n}, \%)$ & & & & $0.40(0.19,0.62)$ & $<0.001$ \\
\hline No & $311(80.36 \%)$ & $226(85.61 \%)$ & $85(69.11 \%)$ & & \\
\hline Yes & $76(19.64 \%)$ & $38(14.39 \%)$ & $38(30.89 \%)$ & & \\
\hline Primary diagnosis (n, \%) & & & & $0.25(0.03,0.47)$ & 0.377 \\
\hline Orthopedic disorders and fractures & 116 (30.61\%) & 81 (3I.40\%) & 35 (28.93\%) & & \\
\hline Cancer & $29(7.65 \%)$ & 21 (8.14\%) & $8(6.61 \%)$ & & \\
\hline Stroke & $4 \mathrm{I}(10.82 \%)$ & 29 (11.24\%) & $12(9.92 \%)$ & & \\
\hline Hypertension and lung disease & $30(7.92 \%)$ & 21 (8.14\%) & $9(7.44 \%)$ & & \\
\hline Phlebitis & $83(21.90 \%)$ & $48(18.60 \%)$ & 35 (28.93\%) & & \\
\hline Other & $80(21.11 \%)$ & $58(22.48 \%)$ & $22(18.18 \%)$ & & \\
\hline Urinary tract infection $(\mathrm{n}, \%)$ & & & & $0.28(0.07,0.50)$ & 0.003 \\
\hline No & $376(97.16 \%)$ & 261 (98.86\%) & II 5 (93.50\%) & & \\
\hline Yes & II (2.84\%) & $3(1.14 \%)$ & $8(6.50 \%)$ & & \\
\hline
\end{tabular}

(Continued) 
Table I (Continued).

\begin{tabular}{|c|c|c|c|c|c|}
\hline Calf Circumference & Overall & $\mathrm{CC} \geq 3 \mathrm{I} \mathrm{cm}$ & $\mathrm{CC}<3 \mathrm{Icm}$ & Standardize Diff. & $P$-value \\
\hline Smoking (n, \%) & & & & $0.02(-0.19,0.23)$ & 0.982 \\
\hline Never & $300(77.52 \%)$ & $204(77.27 \%)$ & 96 (78.05\%) & & \\
\hline Current & $51(13.18 \%)$ & 35 (13.26\%) & $16(13.01 \%)$ & & \\
\hline Former & $36(9.30 \%)$ & $25(9.47 \%)$ & II (8.94\%) & & \\
\hline Surgery (n, \%) & & & & $0.13(-0.09,0.34)$ & 0.248 \\
\hline No & 166 (42.89\%) & 108 (40.91\%) & $58(47.15 \%)$ & & \\
\hline Yes & 221 (57.11\%) & 156 (59.09\%) & $65(52.85 \%)$ & & \\
\hline Bed-rest days (IQR) & $11.00(5.00-18.50)$ & $10.00(5.00-17.00)$ & $13.00(7.00-24.00)$ & $0.27(0.06,0.49)$ & 0.003 \\
\hline Charlson Comorbidity (n, \%) Index & & & & $0.12(-0.10,0.33)$ & 0.285 \\
\hline$<2$ & $266(68.73 \%)$ & $186(70.45 \%)$ & $80(65.04 \%)$ & & \\
\hline$\geq 2$ & 140 (36.18\%) & 78 (29.55\%) & $43(34.96 \%)$ & & \\
\hline Antibiotics (n, \%) & & & & $0.23(0.01,0.44)$ & 0.045 \\
\hline No & $94(24.29 \%)$ & $72(27.27 \%)$ & $22(17.89 \%)$ & & \\
\hline Yes & $293(75.71 \%)$ & $192(72.73 \%)$ & 101 (82.11\%) & & \\
\hline Glucocorticoids (n, \%) & & & & $0.31(0.09,0.52)$ & 0.004 \\
\hline No & 247 (63.82\%) & $18 \mid(68.56 \%)$ & 66 (53.66\%) & & \\
\hline Yes & 140 (36.27\%) & $83(31.44 \%)$ & 57 (46.34\%) & & \\
\hline 90-day mortality (n, \%) & & & & $0.35(0.13,0.56)$ & $<0.001$ \\
\hline No & 366 (94.57\%) & 257 (97.35\%) & 109 (88.62\%) & & \\
\hline Yes & $2 \mathrm{I}(5.43 \%)$ & 7 (2.65\%) & $14(11.38 \%)$ & & \\
\hline
\end{tabular}

Abbreviations: IQR, interquartile range; $C C$, calf circumference.

was $12.66 \%$ for illiterate, $27.65 \%$ for primary school education, and $44.19 \%$ for middle school and above. For complete blood count at hospital admission, the average red blood cell count was 3.91; hemoglobin was $115.90 \mathrm{mg} /$ L; white blood cell count was 9.66; neutrophils, 7.57; and leukomonocyte, 1.29. Regarding medication use, 293 (75.71\%) participants were treated with antibiotics, and $140(36.27 \%)$ participants used glucocorticoids for treatment.

The percentage of those with a urinary tract infection, pneumonia and who were female was significantly higher in the low CC group than in the normal CC group (all $\mathrm{p}<0.05$ ). Additionally, the rate of 90-day mortality was higher in the low CC group than the normal CC group, with a significant difference $(11.38 \%$ versus $2.65 \%$, $P<0.001)$. Those with low CC were likelier to be older in age; and to have lower albumin and BMI (all $P<0.05$ ). There were significant differences between the low $\mathrm{CC}$ group and the normal $\mathrm{CC}$ group in terms of medications (antibiotics and glucocorticoids, both $P<0.05$ ). However, there was no significant difference between these two groups in terms of red and white blood cell count, neutrophils, leukomonocyte, fasting plasma glucose levels, ethnicity, bedsores, being a smoker, having had surgery, primary diagnosis and Charlson Comorbidity Index score.

\section{Univariate Regression Analysis}

Table 2 shows the univariate regression analysis between the different variables and 90-day mortality. The results showed that age, pneumonia, Charlson Comorbidity Index $(\geq 2)$, glucocorticoids and fasting plasma glucose levels were significantly increased risk factors for 90-day mortality. However, albumin, BMI, leukomonocyte, surgery and calf circumference were significant protective factors for mortality. In addition, when we categorized calf circumference into low $\mathrm{CC}(<31 \mathrm{~cm})$ and normal $\mathrm{CC}(\geq 31 \mathrm{~cm})$, it indicated that patients with low $\mathrm{CC}$ had an incremental risk of 90-day mortality $(\mathrm{P}=0.0011)$.

\section{The Relationship Between CC, Albumin, BMI, and Low CC for 90-Day Mortality}

The results of multivariate regression analysis in an unadjusted model indicated that albumin (OR=0.86;95\%CI:0.79-0.94), $\mathrm{CC}(\mathrm{OR}=0.75,95 \% \mathrm{CI}: 0.65-0.86)$ and $\mathrm{BMI}(\mathrm{OR}=0.85 ; 95 \%$ 
Table 2 Univariate Regression Analysis of the Variables Associated with 90-Day Mortality

\begin{tabular}{|c|c|c|c|}
\hline & Statistics & 90-Day Mortality & $P$-value \\
\hline \multicolumn{4}{|l|}{ Gender (n, \%) } \\
\hline Male & 199 (5I.42\%) & I.0 Reference & \\
\hline Female & 188 (48.58\%) & $1.17(0.49,2.83)$ & 0.7202 \\
\hline Age & $61.22 \pm 15.18$ & $1.07(1.03,1.11)$ & 0.0004 \\
\hline \multicolumn{4}{|l|}{ Ethnicity (n, \%) } \\
\hline Han & 369 (95.35\%) & I.0 Reference & \\
\hline Other & $18(4.65 \%)$ & $1.03(0.13,8.11)$ & 0.9802 \\
\hline \multicolumn{4}{|l|}{ Education (n, \%) } \\
\hline Illiterate & 49 (12.66\%) & I.0 Reference & \\
\hline Primary & $107(27.65 \%)$ & $0.43(0.12,1.57)$ & 0.2011 \\
\hline Middle school and above & 171 (44.19\%) & $0.49(0.16,1.53)$ & 0.2197 \\
\hline College degree or above & $60(15.50 \%)$ & $0.30(0.06,1.64)$ & 0.1656 \\
\hline \multicolumn{4}{|l|}{ Primary diagnosis (n, \%) } \\
\hline Orthopedic disorders and fractures & $116(30.61 \%)$ & I.0 reference & \\
\hline Cancer & $29(7.65 \%)$ & $0.56(0.07-4.7 I)$ & 0.5903 \\
\hline Stroke & $41(10.82 \%)$ & $0.39(0.05-3.26)$ & 0.3845 \\
\hline Hypertension and lung disease & 30 (7.92\%) & $2.40(0.65,8.80)$ & 0.1881 \\
\hline Phlebitis & $83(21.90 \%)$ & $0.79(0.22-2.79)$ & 0.7120 \\
\hline Other & $80(21.11 \%)$ & $0.82(0.23-2.90)$ & 0.7574 \\
\hline \multicolumn{4}{|l|}{ Bedsores (n, \%) } \\
\hline No & $369(95.35 \%)$ & I.0 Reference & \\
\hline Yes & $18(4.65 \%)$ & $1.03(0.13,8.11)$ & 0.9802 \\
\hline \multicolumn{4}{|l|}{ Pneumonia (n, \%) } \\
\hline No & $311(80.36 \%)$ & I.0 Reference & \\
\hline Yes & 76 (19.64\%) & $5.09(2.08,12.50)$ & 0.0004 \\
\hline \multicolumn{4}{|l|}{ Urinary tract infection $(\mathrm{n}, \%)$} \\
\hline No & $376(97.16 \%)$ & I.0 Reference & \\
\hline Yes & II (2.84\%) & $4.18(0.84,20.68)$ & 0.0800 \\
\hline Albumin & $35.38 \pm 6.00$ & $0.87(0.80,0.94)$ & 0.0007 \\
\hline BMI (SD, kg/m²) & $23.98 \pm 3.55$ & $0.85(0.74,0.97)$ & 0.0178 \\
\hline Red blood cell (SD, I0^9/L) & $3.91 \pm 1.58$ & $0.71(0.39,1.30)$ & 0.2674 \\
\hline Hemoglobin (g/L, SD) & $115.90 \pm 22.78$ & $0.99(0.97,1.01)$ & 0.3325 \\
\hline White blood cell (SD, I0^9/L) & $9.66 \pm 4.33$ & $1.03(0.94,1.14)$ & 0.5088 \\
\hline Neutrophils (SD, I0^9/L) & $7.57 \pm 4.14$ & $1.07(0.98,1.18)$ & 0.1431 \\
\hline Leukomonocyte (SD, I0^9/L) & $1.29 \pm 0.92$ & $0.13(0.04,0.4 I)$ & 0.0006 \\
\hline \multicolumn{4}{|l|}{ Smoker (n, \%) } \\
\hline Never & $300(77.52 \%)$ & I.0 Reference & \\
\hline Current & $5 \mathrm{I}(13.18 \%)$ & $0.78(0.17,3.50)$ & 0.7407 \\
\hline Former & $36(9.30 \%)$ & $2.37(0.74,7.59)$ & 0.1445 \\
\hline Fasting plasma glucose & $6.85 \pm 2.51$ & $1.24(1.06,1.46)$ & 0.0073 \\
\hline \multicolumn{4}{|l|}{ Surgery (n, \%) } \\
\hline No & $166(42.89 \%)$ & I.0 Reference & \\
\hline Yes & 221 (57.11\%) & $0.22(0.08,0.6 \mathrm{I})$ & 0.0035 \\
\hline
\end{tabular}

(Continued) 
Table 2 (Continued).

\begin{tabular}{|c|c|c|c|}
\hline & Statistics & 90-Day Mortality & $P$-value \\
\hline $\mathrm{CC}(\mathrm{SD}, \mathrm{cm})$ & $32.57 \pm 3.96$ & $0.75(0.66,0.86)$ & $<0.0001$ \\
\hline \multicolumn{4}{|l|}{ CC (n, \%) } \\
\hline$\geq 3 \mathrm{Icm}$ & $264(68.22 \%)$ & I.0 Reference & \\
\hline$<31 \mathrm{~cm}$ & 123 (31.78\%) & $4.72(1.85,12.01)$ & 0.0011 \\
\hline Bed-rest days (IQR) & $11.00(5.00-18.50)$ & $1.02(1.00,1.04)$ & 0.0549 \\
\hline \multicolumn{4}{|l|}{ Charlson Comorbidity Index (n, \%) } \\
\hline$<2$ & $266(68.73 \%)$ & I.0 reference & \\
\hline$\geq 2$ & $121(3 \mid .27 \%)$ & $7.95(2.84,22.27)$ & $<0.0001$ \\
\hline \multicolumn{4}{|l|}{ Antibiotics (n, \%) } \\
\hline No & 94 (24.29\%) & I.0 Reference & \\
\hline Yes & $293(75.71 \%)$ & $3.19(0.73,13.96)$ & 0.1235 \\
\hline \multicolumn{4}{|l|}{ Glucocorticoids (n, \%) } \\
\hline No & 247 (63.82\%) & I.0 Reference & \\
\hline Yes & 142 (36.18\%) & $3.06(1.24,7.57)$ & 0.0157 \\
\hline
\end{tabular}

Abbreviation: CC, calf circumference.

CI:0.74-0.97) were protective factors for mortality, whereas low $\mathrm{CC}(\mathrm{OR}=4.71 ; 95 \% \mathrm{CI}: 1.85-12.00)$ was a risk factor for mortality. These associations were still unchanged after adjusting for age, gender, and education, with the exception of BMI ( $\mathrm{OR}=0.89 ; 95 \% \mathrm{CI}: 0.78-1.02)$. However, after adjusting the completed variables (age, gender, education, ethnicity, smoking, red blood cell count, hemoglobin; white blood cell count, neutrophils, leukomonocyte, pneumonia, urinary tract infection, Charlson Comorbidity Index $(\geq 2)$, antibiotics, glucocorticoids and surgery), only $\mathrm{CC}(\mathrm{OR}=0.79,95 \% \mathrm{CI}: 0.65-0.96$, $P=0.017)$ was still a protective factor for mortality, together with low $\mathrm{CC}(\mathrm{OR}=4.24,95 \% \mathrm{CI}: 1.07-16.74 P=0.038)$ being a risk factor for mortality. All detailed information is displayed in Table 3.

\section{Non-Linear Relationship Analyses}

The association between $\mathrm{CC}$ and morality was analyzed with a generalized additive model (GAM), and the results showed that with an increase in $\mathrm{CC}$, the mortality rate decreased. (Figure 1)

\section{Prognostic Score Comparison}

Receiver operating characteristic curve analysis was conducted to compare the performance of different models in

Table 3 Multivariate Regression Analysis Between Nutritional Status (CC, Albumin, and BMI) and Mortality in Different Models

\begin{tabular}{|c|c|c|c|c|c|c|}
\hline Exposure & Non-Adjusted (OR, 95\% Cl) & P-value & Adjusted I (OR, 95\% CI) & P-value & Adjusted II (OR, 95\% CI) & P-value \\
\hline BMI & $0.85(0.74,0.97)$ & 0.017 & $0.89(0.78,1.02)$ & 0.088 & $0.89(0.76,1.05)$ & 0.185 \\
\hline $\mathrm{CC}(\mathrm{Icm})$ & $0.75(0.65,0.86)$ & $<0.001$ & $0.79(0.68,0.92)$ & 0.002 & $0.79(0.65,0.96)$ & 0.017 \\
\hline Albumin & $0.86(0.79,0.94)$ & 0.0006 & $0.86(0.79,0.95)$ & 0.003 & $0.94(0.83,1.07)$ & 0.390 \\
\hline \multicolumn{7}{|l|}{$C C(n, \%)$} \\
\hline$\geq 3 \mathrm{Icm}$ & I.0 Reference & & I.0 Reference & & I.0 Reference & \\
\hline$<31 \mathrm{~cm}$ & $4.71(1.85,12.00)$ & 0.001 & $3.59(1.32,9.74)$ & 0.011 & $4.24(1.07,16.74)$ & 0.038 \\
\hline
\end{tabular}

Notes: Exposure: CC; CC category; albumin; BMI. Non-adjusted model. Adjusted I model adjusted for: age, gender, education. Adjusted II model adjusted for: age; gender; education; ethnicity; smoking; red blood cell; hemoglobin; white blood cell; neutrophils; leukomonocyte; pneumonia; urinary tract infection; surgery, antibiotics, glucocorticoids, Charlson Comorbidity Index.

Abbreviation: CC, calf circumference. 


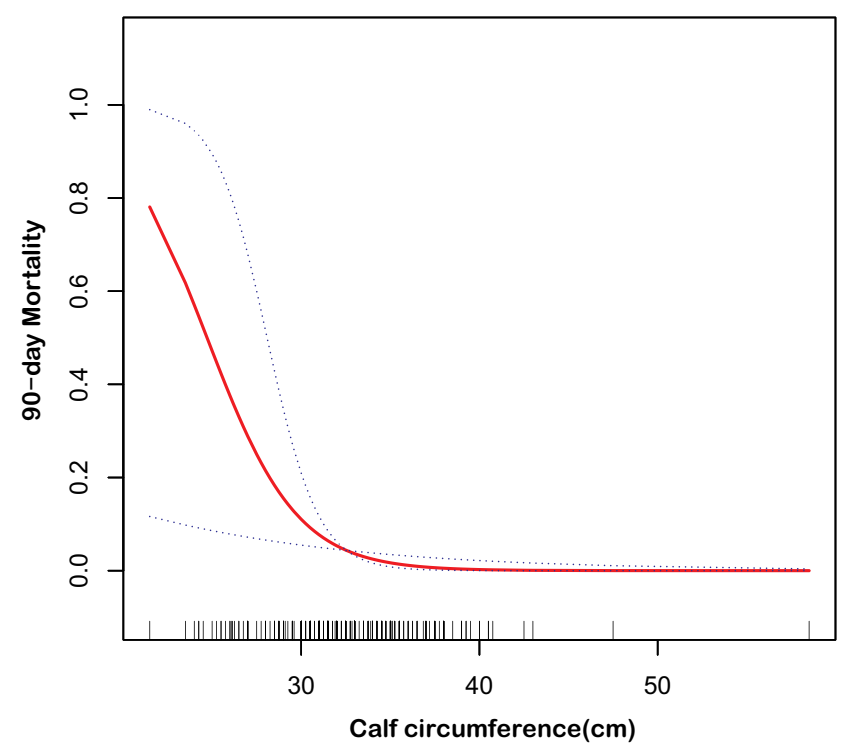

Figure I A nonlinear relationship between calf circumference and rate of 90 -day mortality using a generalized additive model.

predicting mortality (Figure 2). The results found that the AUC of CC, albumin, and BMI for predicting 90-day mortality was $0.759 \quad(95 \% \mathrm{CI}: 0.662-0.856), \quad 0.735 \quad(95 \%$ CI:0.638-0.833) and 0.653 (95\%CI:0.538-0.767), respectively. The AUC of CC ( $\mathrm{p}=0.0336)$ was higher than BMI, with statistically significant differences. However, the AUC difference between albumin and BMI was not statistically significant $(\mathrm{P}=0.25)$. Moreover, when combining albumin and $\mathrm{CC}$, discrimination was improved with the $\mathrm{AUC}=0.812$

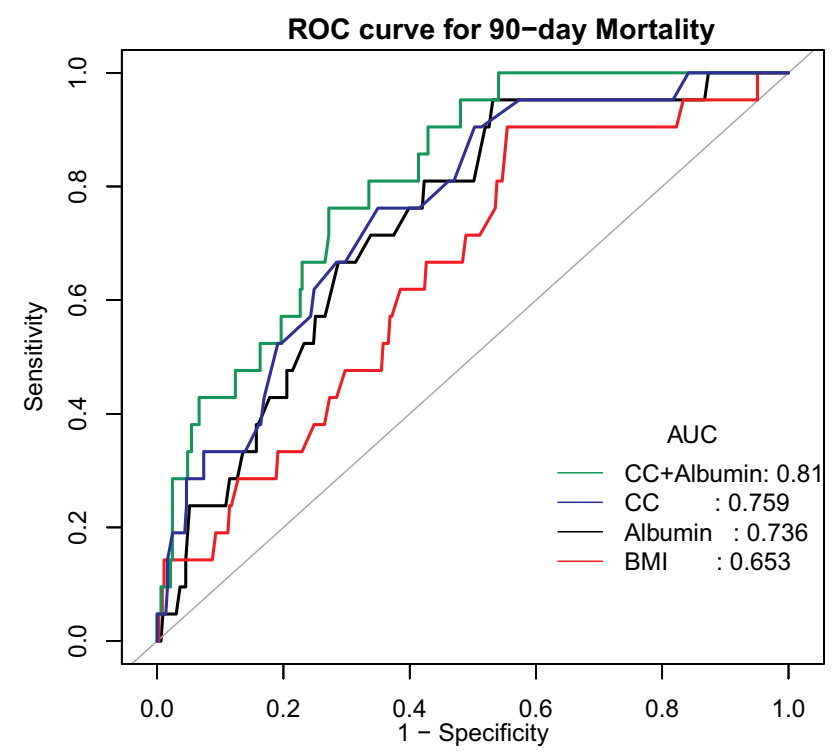

Figure 2 The ROC curve analysis for various models, based on different variables: Model I for combined calf circumference and albumin (green); Model 2: for calf circumference (Blue); Model 3: for albumin (Black); Model 4: for BMI(Red).
(95\% CI $=0.733-0.890)$, which was higher than BMI $(\mathrm{P}=0.001)$ (Table 4).

\section{Discussion}

Our study indicates that immobile patients with low CC are at increased risk of mortality, compared to those with normal $\mathrm{CC}$; and $\mathrm{CC}$ value was a protective factor for mortality. In addition, our study demonstrates that CC performance discrimination was better than BMI in predicting 90-day mortality. When CC was combined with albumin, its discrimination performance was improved, compared to BMI. This finding indicates that combined albumin and $\mathrm{CC}$ had relatively good predictive discrimination ( $\mathrm{ROC}=0.812$ ) for predicting 90-day mortality, and by using this tool, we can proactively stratify high-risk groups and carry out effective interventions to improve the outcomes of immobile inpatients.

Immobile patients often suffer from malnutrition, attributed to multiple comorbidities. They present in a high level of a catabolic state, which leads to loss of protein from muscle. ${ }^{34}$ In our study, we found that immobile patients with low CC had a higher likelihood of being older in age, and suffering from pneumonia and urinary tract infection. Therefore, immobile inpatients who are older with comorbidities were a high-risk group that needed nutrition screening. BMI was considered a parameter index to assess inpatient nutritional status, with unsatisfactory application, because BMI fails to consistently distinguish between lean and obese patients. ${ }^{35}$ As we all know, BMI is reported to have a " $U$ " or "J" relationship with mortality in different populations. ${ }^{36,37}$ In our study, BMI was a protective factor for mortality in an unadjusted model; however, after adjusting a variety of variables, this association was found not to exist, indicating that BMI is not a stable prognostic factor for mortality. In fact, BMI cannot completely reflect nutritional status, especially in older inpatients, because it does not precisely possess an indication of total body muscle mass. ${ }^{38}$ A number of studies demonstrate that many patients with adequate BMI also present with low muscle mass, ${ }^{39,40}$ meaning that we should be cautious in using BMI alone to assess nutritional risk. Easton et $\mathrm{al}^{22}$ have stated that BMI is a poor indicator for predicting patients dying with frailty. A similar viewpoint was confirmed by Tsai et al. ${ }^{20}$

Our findings indicate that immobile patients with low $\mathrm{CC}$ can have an incremental risk of mortality, compared with patients with normal $\mathrm{CC}$, which was in line with numerous studies. ${ }^{19,21,22}$ Sousa et $\mathrm{al}^{19}$ conducted a study 
Table 4 Prediction Performance of Four Variables for Estimating 90-Day Mortality

\begin{tabular}{|l|l|l|l|l|}
\hline & CC+Albumin & CC & Albumin & BMI \\
\hline AUC (95\% Cl) & $0.812(0.733-0.890)$ & $0.759(0.662-0.856)$ & $0.735(0.638-0.833)$ & $0.653(0.538-0.767)$ \\
\hline P-value (vs BMI) & 0.001 & 0.033 & 0.250 & - \\
\hline Cutoff & -0.275 & 31.125 & 36.35 & 24.515 \\
\hline Sensitivity \% & 0.728 & 0.650 & 0.466 & 0.445 \\
\hline Specificity \% & 0.761 & 0.761 & 0.952 & 0.904 \\
\hline PPV & 0.150 & 0.111 & 0.102 & 0.085 \\
\hline NPV & 0.979 & 0.979 & 0.993 & 0.987 \\
\hline PLR & 2.802 & 2.802 & 1.785 & 1.631 \\
\hline NLR & 0.327 & 0.327 & 0.102 & 0.213 \\
\hline
\end{tabular}

Abbreviation: CC, calf circumference.

of 250 cancer patients and found low $\mathrm{CC}$ was an independent predictor of mortality, by using a cutoff value of $<34 \mathrm{~cm}$ for men and $<33 \mathrm{~cm}$ for women $(\mathrm{HR}=3.01 ; 95 \%$ $\mathrm{CI}=1.52-5.98)$. Another study also reported similar results $(\mathrm{HR}=3.39 ; 95 \% \mathrm{CI}: 1.80-6.39)$ with a cutoff of $<31 \mathrm{~cm}$ for both women and men. ${ }^{21}$ Recently, the cutoff value of defining low $\mathrm{CC}$ has varied, with some studies using $31 \mathrm{~cm}$, while other studies used $<28 \mathrm{~cm}^{30}$ On the other hand, some studies used the standard definition of sarcopenia ( $<34 \mathrm{~cm}$ for men and $<33 \mathrm{~cm}$ for women). ${ }^{31}$ We suspect this is partly the reason that inconsistent results were generated when using different cutoff values.

The mechanism for the association between muscle mass and mortality is complicated but widely reported. Low muscle mass is a core component of defining sarcopenia that is substantially associated with increased risk of mortality in various populations, such as communitydwelling older adults, ${ }^{22}$ nursing home residents ${ }^{41}$ or hospitalized patients. ${ }^{42}$ To sum up, skeletal muscle is the fundamental basis for human ability to maintain locomotion and respiration. ${ }^{43}$ Declining muscle mass embodies worsening health and can be attributed to multiple chronic diseases, which in turn results in a weakened immune system and a series of comorbidities, ${ }^{44,45}$ eventually increasing the risk of mortality. However, multiple interventions, such as nutrition and resistance exercises, can delay disease progression and to some extent reverse this condition. ${ }^{46}$ Therefore, screening and identifying low $\mathrm{CC}$ is essential for improving patient clinical outcomes.
The AUC of CC in predicting 90-day mortality was 0.759, which was higher than BMI $(\mathrm{P}=0.033)$. A previous study also found that a TPA parameter that indicates muscle mass using CT scan has a good discrimination performance in predicting ICU mortality (AUROC $=0.702 ; 95 \% \mathrm{CI}: 0.555-0.849$ ), which was relatively lower than our result $\left(\right.$ AUROC $=0.759 ; 95 \%$ CI:0.662-0.855). ${ }^{47}$ Similarly, Ng et $\mathrm{al}^{48}$ reported that SMI performance for predicting hospital mortality among critically ill patients was relatively moderate $(\mathrm{AUC}=0.637)$. Apart from performance, $\mathrm{CC}$ has various advantages, such as being simple, convenient and inexpensive, compared to medical imaging methods. In addition, when we combined $\mathrm{CC}$ with albumin, the AUC of performance discrimination for predicting mortality was $0.812(95 \% \mathrm{CI}=0.733-0.890)$ with sensitivity being 0.762 and specificity being 0.728 , indicating this predictive model performs relatively well in predicting mortality risk in immobile patients.

Our study has multiple clinical implications. First, there is an urgent need to estimate the risk of mortality among immobile patients, generating an opportunity to produce a simple and verified model system to help medical staff create a comprehensive treatment therapy. Our study has demonstrated that this new predictive model can meet these clinical needs. Second, this predictive model only used two parameters, albumin and $\mathrm{CC}$, which can very easily be secured in a busy and complicated clinical setting. Rather than assess muscle mass by using imaging 
equipment, medical staff can acquire these parameters using a convenient method that is both economical and not time-consuming.

\section{Limitations}

However, we need to be cautious in interpreting these findings. First, the sample size in our study was not large enough, which may have had a potential impact on our results. Second, we did not perform nutritional assessments, such as Nutritional risk (NRS-2002); thus we cannot estimate the association between $\mathrm{CC}$ and nutritional risk score. Third, we cannot completely overlook a potential error, in that some inpatients may have had possible symptoms of edema, leading to an overestimation of $\mathrm{CC}$ value. Fourth, other important factors, such as different treatment programs, may also have influenced inpatient outcomes. Fifth, our study might have had some selective bias because we only included participants who had completed CC measurements, which is not random sampling. As shown in the supplemental files (Tables

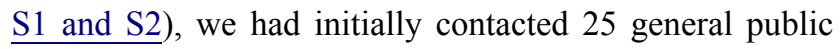
hospitals. However, our study was a secondary analysis that only included immobile patients whose calf circumference was measured. As a result, 12 hospitals were excluded, resulting in 13 hospitals (6 tertiary hospitals, $\mathrm{n}=358$ and 7 non-tertiary hospitals, $\mathrm{n}=29$ ) that were included for data analysis. Given that the majority of participants $(92.5 \%)$ came from tertiary hospitals, future studies with larger samples from different levels of hospital should be conducted to verify our results. Sixth, the patients we recruited did have different primary diagnoses. In our final logistic regression model, the result showed that low $\mathrm{CC}$ was associated with a high risk of mortality after adjusting Charlson Comorbidity Index. That said, we still cannot totally exclude the possible effect of the disease itself on mortality. Although our study has some limitations, it also has several strengths that merit clinical application. For example, we used comprehensive statistical methods, such as generalized additive model (GAM) and adjusted completed variables in multivariate analysis. Second, to the best of our knowledge, this is the first study to compare the performance of $\mathrm{CC}$, albumin and BMI to predict mortality in immobile inpatients, which establishes a new predictive model for mortality in immobile patients. Third, we believe that our results can help clinicians to identify immobile patients who are at high risk. More studies with a larger sample size to investigate this important issue will be warranted in the future.

\section{Conclusions}

Our study shows that the relationship between calf circumference and 90-day mortality is significantly negative. In addition, immobile patients with a low calf circumference have about a four-fold risk of 90-day mortality, compared to patients with normal low calf circumference. We also found that calf circumferences combined with albumin had a good discrimination in predicting mortality in immobile patients. This finding could, in brief, help clinical staff evaluate high-risk groups for mortality early on and perform effective interventions, such as nutritional recommendations and resistance exercises designed to improve muscle mass.

\section{Acknowledgments}

We sincerely thank all of the inpatients for their participation and understanding. We also thank the Department of Nursing, Peking Union Medical College, for its support.

\section{Ethical Standards Disclosure}

The project was approved by the Ethics Committee of Peking Union Medical College Hospital (S-700). Written informed participant consent forms were obtained prior to study commencement. The authors confirm that this study was conducted in accordance with the Declaration of Helsinki.

\section{Funding}

The National Health Commission of the People's Republic of China funded this study with grant number 201502017.

\section{Disclosure}

The authors reported no conflicts of interest for this work.

\section{References}

1. Barker LA, Gout BS, Crowe TC. Hospital malnutrition: prevalence, identification and impact on patients and the healthcare system. Int J Environ Res Public Health. 2011;8(2):514-527. doi:10.3390/ ijerph8020514

2. Konturek PC, Herrmann HJ, Schink K, Neurath MF, Zopf Y. Malnutrition in hospitals: it was, is now, and must not remain a problem! Med Sci Monit. 2015;21:2969-2975. doi:10.12659/ MSM.894238

3. Agarwal E, Miller M, Yaxley A, Isenring E. Malnutrition in the elderly: a narrative review. Maturitas. 2013;76(4):296-302. doi:10.1016/j.maturitas.2013.07.013

4. Corkins MR, Guenter P, DiMaria-Ghalili RA, et al. Malnutrition diagnoses in hospitalized patients: United States, 2010. JPEN $J$ Parenter Enteral Nutr. 2014;38(2):186-195. doi:10.1177/ 0148607113512154 
5. Norman K, Pichard C, Lochs H, Pirlich M. Prognostic impact of disease-related malnutrition. Clin Nutr. 2008;27(1):5-15. doi:10.1016/j.clnu.2007.10.007

6. Liu H, Jiao J, Zhu C, et al. Associations between nutritional status, sociodemographic characteristics, and health-related variables and health-related quality of life among Chinese elderly patients: a Multicenter Prospective Study. Front Nutr. 2020;7:583161. doi:10.3389/fnut.2020.583161

7. Correia MI, Waitzberg DL. The impact of malnutrition on morbidity, mortality, length of hospital stay and costs evaluated through a multivariate model analysis. Clin Nutr. 2003;22(3):235-239. doi:10.1016/S0261-5614(02)00215-7

8. Cederholm T, Jensen GL, Correia M, et al. GLIM criteria for the diagnosis of malnutrition - a consensus report from the global clinical nutrition community. Clin Nutr. 2019;38(1):1-9. doi:10.1016/j. clnu.2018.08.002

9. Lidoriki I, Schizas D, Mpaili E, et al. Associations between skeletal muscle mass index, nutritional and functional status of patients with oesophago-gastric cancer. Clin Nutr ESPEN. 2019;34:61-67. doi:10.1016/j.clnesp.2019.08.012

10. Cruz-Jentoft AJ, Sayer AA. Sarcopenia. Lancet. 2019;393 (10191):2636-2646. doi:10.1016/S0140-6736(19)31138-9

11. Cretoiu SM, Zugravu CA. Nutritional considerations in preventing muscle atrophy. Adv Exp Med Biol. 2018;1088:497-528.

12. Cruz-Jentoft AJ, Bahat G, Bauer J, et al. Sarcopenia: revised European consensus on definition and diagnosis. Age Ageing. 2019;48(4):601. doi:10.1093/ageing/afz046

13. Miyake H, Kanazawa I, Tanaka KI, Sugimoto T. Low skeletal muscle mass is associated with the risk of all-cause mortality in patients with type 2 diabetes mellitus. Ther Adv Endocrinol Metab. 2019;10:2042018819842971. doi:10.1177/2042018819842971

14. Baggerman MR, van Dijk DPJ, Winkens B, et al. Muscle wasting associated comorbidities, rather than sarcopenia are risk factors for hospital mortality in critical illness. J Crit Care. 2020;56:31-36. doi:10.1016/j.jcrc.2019.11.016

15. Landi F, Camprubi-Robles M, Bear DE, et al. Muscle loss: the new malnutrition challenge in clinical practice. Clin Nutr. 2019;38 (5):2113-2120. doi:10.1016/j.clnu.2018.11.021

16. Beaudart $\mathrm{C}$, McCloskey E, Bruyère $\mathrm{O}$, et al. Sarcopenia in daily practice: assessment and management. BMC Geriatr. 2016;16 (1):170. doi:10.1186/s12877-016-0349-4

17. Kawakami R, Murakami H, Sanada K, et al. Calf circumference as a surrogate marker of muscle mass for diagnosing sarcopenia in Japanese men and women. Geriatr Gerontol Int. 2015;15 (8):969-976. doi:10.1111/ggi.12377

18. Kawakami R, Miyachi M, Sawada SS, et al. Cutoffs for calf circumference as a screening tool for low muscle mass: WASEDA'S Health Study. Geriatr Gerontol Int. 2020;20(10):943-950. doi:10.1111/ggi.14025

19. Sousa IM, Bielemann RM, Gonzalez MC, et al. Low calf circumference is an independent predictor of mortality in cancer patients: a Prospective Cohort Study. Nutrition. 2020;79-80:110816. doi:10.1016/j.nut.2020.110816

20. Tsai AC, Lai MC, Chang TL. Mid-arm and calf circumferences (MAC and CC) are better than body mass index (BMI) in predicting health status and mortality risk in institutionalized elderly Taiwanese. Arch Gerontol Geriatr. 2012;54(3):443-447. doi:10.1016/j. archger.2011.05.015

21. Valmorbida E, Trevisan C, Imoscopi A, Mazzochin M, Manzato E, Sergi G. Malnutrition is associated with increased risk of hospital admission and death in the first 18 months of institutionalization. Clin Nutr. 2020;39(12):3687-3694. doi:10.1016/j.clnu.2020.03.029

22. Easton JF, Stephens CR, Román-Sicilia H, Cesari M, PérezZepeda MU. Anthropometric measurements and mortality in frail older adults. Exp Gerontol. 2018;110:61-66. doi:10.1016/j. exger.2018.05.011
23. Rodrigues J, Santin F, Brito F, Lindholm B, Stenvinkel P, Avesani CM. Nutritional status of older patients on hemodialysis: which nutritional markers can best predict clinical outcomes? Nutrition. 2019;65:113-119. doi:10.1016/j.nut.2019.03.002

24. Tarnowski M, Stein E, Marcadenti A, Fink J, Rabito E, Silva FM. Calf circumference is a good predictor of longer hospital stay and nutritional risk in emergency patients: a Prospective Cohort Study. J Am Coll Nutr. 2020;39(7):645-649. doi:10.1080 07315724.2020 .1723452

25. de Almeida Roediger M, de Fátima Nunes Marucci M, Quintiliano Scarpelli Dourado DA, de Oliveira C, Licio Ferreira Santos J, de Oliveira Duarte YA. Body composition changes and 10-year mortality risk in older brazilian adults: analysis of prospective data from the SABE Study. J Nutr Health Aging. 2019;23(1):51-59. doi:10.1007/ s12603-018-1118-1

26. de Sousa OV, Mendes J, Amaral TF. Nutritional and functional indicators and their association with mortality among older adults with Alzheimer's disease. Am J Alzheimers Dis Other Demen. 2020;35:1533317520907168. doi:10.1177/1533317520907168

27. Yin L, Lin X, Li N, et al. Evaluation of the global leadership initiative on malnutrition criteria using different muscle mass indices for diagnosing malnutrition and predicting survival in lung cancer patients. JPEN J Parenter Enteral Nutr. 2020. doi:10.1002/ jpen. 1873

28. Ho SC, Wang JY, Kuo HP, et al. Mid-arm and calf circumferences are stronger mortality predictors than body mass index for patients with chronic obstructive pulmonary disease. Int J Chron Obstruct Pulmon Dis. 2016;11:2075-2080. doi:10.2147/COPD.S107326

29. Teixeira PP, Kowalski VH, Valduga K, de Araújo BE, Silva FM. Low muscle mass is a predictor of malnutrition and prolonged hospital stay in patients with acute exacerbation of chronic obstructive pulmonary disease: a Longitudinal Study. JPEN J Parenter Enteral Nutr. 2020. doi:10.1002/jpen.1998

30. Moon S, Hong GS. Predictive factors of mortality in older adult residents of long-term care facilities. J Nurs Res. 2020;28(2):e82. doi:10.1097/jnr.0000000000000356

31. Aliberti MJR, Szlejf C, Covinsky KE, Lee SJ, Jacob-Filho W, Suemoto CK. Prognostic value of a rapid sarcopenia measure in acutely ill older adults. Clin Nutr. 2020;39(7):2114-2120. doi:10.1016/j.clnu.2019.08.026

32. Li J, Wu X, Li Z, et al. Nursing resources and major immobility complications among bedridden patients: a Multicenter Descriptive Study in China. J Nurs Manag. 2019;27(5):930-938. doi:10.1111/ jonm. 12731

33. Cuervo M, Ansorena D, García A, González Martínez MA, Astiasarán I, Martínez JA. [Assessment of calf circumference as an indicator of the risk for hyponutrition in the elderly]. Nutr Hosp. 2009;24(1):63-67. Spanish.

34. Gomes F, Schuetz P, Bounoure L, et al. ESPEN guidelines on nutritional support for polymorbid internal medicine patients. Clin Nutr. 2018;37(1):336-353. doi:10.1016/j.clnu.2017.06.025

35. Weber DR, Leonard MB, Shults J, Zemel BS. A comparison of fat and lean body mass index to BMI for the identification of metabolic syndrome in children and adolescents. J Clin Endocrinol Metab. 2014;99(9):3208-3216. doi:10.1210/jc.2014-1684

36. Thinggaard M, Jacobsen R, Jeune B, Martinussen T, Christensen K. Is the relationship between BMI and mortality increasingly U-shaped with advancing age? A 10-year follow-up of persons aged 70-95 years. J Gerontol a Biol Sci Med Sci. 2010;65(5):526-531. doi:10.1093/gerona/glp214

37. Bhaskaran K, Dos-Santos-Silva I, Leon DA, Douglas IJ, Smeeth L. Association of BMI with overall and cause-specific mortality: a Population-Based Cohort Study of 3.6 million adults in the UK. Lancet Diabetes Endocrinol. 2018;6(12):944-953. doi:10.1016/ S2213-8587(18)30288-2 
38. Müller MJ, Braun W, Enderle J, Bosy-Westphal A. Beyond BMI: conceptual issues related to overweight and obese patients. Obes Facts. 2016;9(3):193-205. doi:10.1159/000445380

39. Martin L, Birdsell L, Macdonald N, et al. Cancer cachexia in the age of obesity: skeletal muscle depletion is a powerful prognostic factor, independent of body mass index. J Clin Oncol. 2013;31 (12):1539-1547. doi:10.1200/JCO.2012.45.2722

40. Batsis JA, Villareal DT. Sarcopenic obesity in older adults: aetiology, epidemiology and treatment strategies. Nat Rev Endocrinol. 2018;14 (9):513-537.

41. Zhang X, Wang C, Dou Q, Zhang W, Yang Y, Xie X. Sarcopenia as a predictor of all-cause mortality among older nursing home residents: a systematic review and meta-analysis. BMJ Open. 2018;8(11): e021252. doi:10.1136/bmjopen-2017-021252

42. Sipers W, de Blois W, Schols J, van Loon LJC, Verdijk LB. Sarcopenia is related to mortality in the acutely hospitalized geriatric patient. J Nutr Health Aging. 2019;23(2):128-137. doi:10.1007/ s12603-018-1134-1

43. Rivas DA, Fielding RA. Skeletal muscle. In: Caballero B, editor. Encyclopedia of Human Nutrition. 3rd ed. Waltham: Academic Press; 2013:193-199.

44. Nelke C, Dziewas R, Minnerup J, Meuth SG, Ruck T. Skeletal muscle as potential central link between sarcopenia and immune senescence. EBioMedicine. 2019;49:381-388. doi:10.1016/j. ebiom.2019.10.034
45. Kalyani RR, Corriere M, Ferrucci L. Age-related and disease-related muscle loss: the effect of diabetes, obesity, and other diseases. Lancet Diabetes Endocrinol. 2014;2(10):819-829. doi:10.1016/S22138587(14)70034-8

46. Li Z, Cui M, Yu K, et al. Effects of nutrition supplementation and physical exercise on muscle mass, muscle strength and fat mass among sarcopenic elderly: a randomized controlled trial. Appl Physiol Nutr Metab. 2020;46(5):494-500. doi:10.1139/apnm-20200643

47. Zhang XM, Zhang WW, Yu XZ, Dou QL, Cheng AS. Comparing the performance of SOFA, TPA combined with SOFA and APACHE-II for predicting ICU mortality in critically ill surgical patients: a secondary analysis. Clin Nutr. 2020;39(9):2902-2909. doi:10.1016/j.clnu.2019.12.026

48. Ng CC, Lee ZY, Chan WY, et al. Low muscularity as assessed by abdominal computed tomography on intensive care unit admission is associated with mortality in a critically ill Asian population. JPEN $J$ Parenter Enteral Nutr. 2020;44(3):425-433. doi:10.1002/jpen.1666
Risk Management and Healthcare Policy

\section{Publish your work in this journal}

Risk Management and Healthcare Policy is an international, peerreviewed, open access journal focusing on all aspects of public health, policy, and preventative measures to promote good health and improve morbidity and mortality in the population. The journal welcomes submitted papers covering original research, basic science, clinical \& epidemiological studies, reviews and evaluations,
Dovepress

guidelines, expert opinion and commentary, case reports and extended reports. The manuscript management system is completely online and includes a very quick and fair peer-review system, which is all easy to use. Visit http://www.dovepress.com/testimonials.php to read real quotes from published authors. 